\title{
On Separation for Multiple Access Channels
}

\author{
Siddharth Ray and Muriel Médard \\ Laboratory for Information and \\ Decision Systems \\ Massachusetts Institute of Technology \\ Cambridge, MA 02139, USA \\ Email: \{sray, medard\}@mit.edu
}

\author{
Michelle Effros \\ Dept. of Electrical Engineering \\ California Institute of Technology \\ Pasadena, CA 91125, USA \\ Email: effros@caltech.edu
}

\author{
Ralf Koetter \\ Coordinated Science Laboratory \\ University of Illinois at \\ Urbana-Champaign \\ Urbana, IL 61801, USA \\ Email:koetter@uiuc.edu
}

\begin{abstract}
We examine the issue of separation for multiple access channels. We demonstrate that source-channel separation holds for noisy multiple access channels, when the channel operates over a common finite field. This robustness of separation is predicated on the fact that noise and inputs are independent, and we examine the loss from failure of separation when noise is input dependent.
\end{abstract}

\section{INTRODUCTION}

The failure of source-channel separation in networks is often considered to be an impediment in applying information theoretic tools in network settings. The simple multiple access channel of Figure 1 gives one example of how separation can fail [1]. The receiver's channel output is the integer sum of the binary channel inputs of $m \geq 2$ users, yielding a channel output alphabet of size $m+1$. Since independent, uniformly distributed input signals fail to achieve the maximum mutual information between the transmitted and received signals, direct transmission of dependent source bits over the channel without channel coding sometimes yields higher achievable transmission rates than Slepian-Wolf source coding followed by multiple access channel coding.

While this simple example may at first appear to estab-

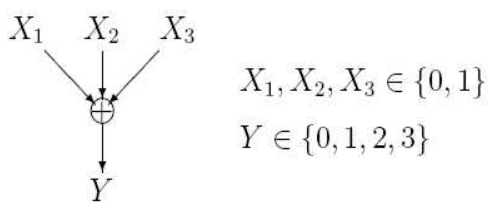

Figure 1: A linear network for which source-channel separation fails [1].

lish irrefutably the failure of source-channel separation in multiple access networks, its simplicity is misleading. In particular, note that the alphabet size of the output is dependent on the number of transmitters. Thus, the network lacks a consistent digital framework. Replacing integer addition with binary addition to give a channel with input and output alphabets of the same cardinality yields a communication system for which separation holds.

In this paper, we argue that source-channel separation is more robust than counterexamples may suggest. We assert, however, that separate source and channel code design does not necessarily simplify the design of communication systems for digital networks. The operations of compression and channel coding are conceptual tools rather than necessary components. While modularity, such as that afforded by the separation theorem, is desirable in the design of components, the decomposition of a problem into modular tasks may increase complexity when the decomposition imposes unnecessary constraints. We treat an important type of canonical network: the noisy multiple access channel, such as may arise in wireless transmissions. Multiple access channels are not only one of the fundamental building blocks of network information theory, but also generally demonstrate the breakdown of separation between source coding and coding over the channel or, rather, network. We show that source-channel separation is optimal for inputindependent noise which may be additive or in the form of erasures. However, separation may fail to achieve the optimal performance when additive noise is inputdependent. For the additive noise channel over the binary field, we compute the maximum difference between the sum channel capacities when channel encoding is done with complete collaboration between the channel encoders and with no collaboration between the channel encoders. We also obtain an expression for the probability that the two sum capacities differ for a binary additive noise multiple access channel picked randomly from the ensemble of all channels of this class.

In section II, we address the topic of source-channel separation for multiple access channels and conclude in section III.

\section{SOURCE-CHANNEL SEPARATION FOR MULTIPLE ACCESS CHANNELS}

It is well known that source-channel separation holds for single-transmitter, single-receiver channels. Thus, the source and channel coding operations can be separated without loss in optimality. We address the topic of separation for source-multiple access channel pairs. Consider a discrete source pair $\left(U_{1}, U_{2}\right)$ with associated rate pair $\left(R_{1}^{U}, R_{2}^{U}\right)$. The Slepian-Wolf region [4] for this source pair is specified as the closure of the convex hull of all rate pairs, $\left(R_{1}^{U}, R_{2}^{U}\right)$, satisfying

$R_{1}^{U}>H\left(U_{1} \mid U_{2}\right), R_{2}^{U}>H\left(U_{2} \mid U_{1}\right), R_{1}^{U}+R_{2}^{U}>H\left(U_{1}, U_{2}\right)$. 
Two transmitters transmit discrete symbols to a single receiver whose received alphabet is also discrete. We denote the channel inputs, their associated rates and output symbol as $\left(X_{1}, X_{2}\right),\left(R_{1}, R_{2}\right)$ and $Y$, respectively.

Let us summarize some known results on multiple access capacity regions. There are three categories of multiple access:

- The most general multiple access is when the channel encoding is done with full collaboration between the channel encoders. Optimal source coding can be performed with or without [4] collaboration between the source encoders. Moreover, there is no loss in optimality in separating the source and channel encoding operations since full collaboration exists between the two channel encoders. We will call this multiple access scheme as "Collaborative Multiple Access" (CMA). The capacity region for this type of multiple access is derived by Liao [3].

- The second type of multiple access is when the source and channel coding at each transmitter is combined into a single operation and there is no collaboration between the joint source-channel encoders at the two transmitters. The encoders directly map the source pairs to channel inputs. We will refer to this multiple access scheme as "Non-collaborative Joint Multiple Access" (NJMA) and the capacity region for this scheme is derived by Cover and El Gamal [2].

- The least general, but most often considered, is multiple access where source and channel coding are performed separately at each transmitter and there is no collaboration between the encoders at the transmitters. We will refer to this multiple access scheme as the "Non-collaborative Separate Multiple Access" (NSMA) and the capacity region for this multiple access scheme is derived by Cover and Wyner $[1,5]$.

The three multiple access capacity schemes are shown in Figure 2. Note that the figure for NSMA is same as Figure 1 in [5]. Reference [7] studies multiple access capacity using graphs as a digital interface between source and channel coding.

CMA capacity region: For this type of multiple access, the channel encoders cooperate with each other and can generate any joint input probability distribution, $P_{X_{1} X_{2}}\left(x_{1}, x_{2}\right)$. For any $P_{X_{1} X_{2}}\left(x_{1}, x_{2}\right)$, denote the closure of the convex hull of all rate pairs $\left(R_{1}, R_{2}\right)$ satisfying

$$
\begin{array}{r}
R_{1}<I\left(X_{1} ; Y \mid X_{2}\right), R_{2}<I\left(X_{2} ; Y \mid X_{1}\right), \\
R_{1}+R_{2}<I\left(X_{1}, X_{2} ; Y\right),
\end{array}
$$

as $\mathbb{L}\left[P_{X_{1} X_{2}}\left(x_{1}, x_{2}\right)\right]$. The CMA capacity region, $\mathbb{R}_{C M A}$, is the convex hull of the sets $\mathbb{L}\left[P_{X_{1} X_{2}}\left(x_{1}, x_{2}\right)\right]$ over all joint
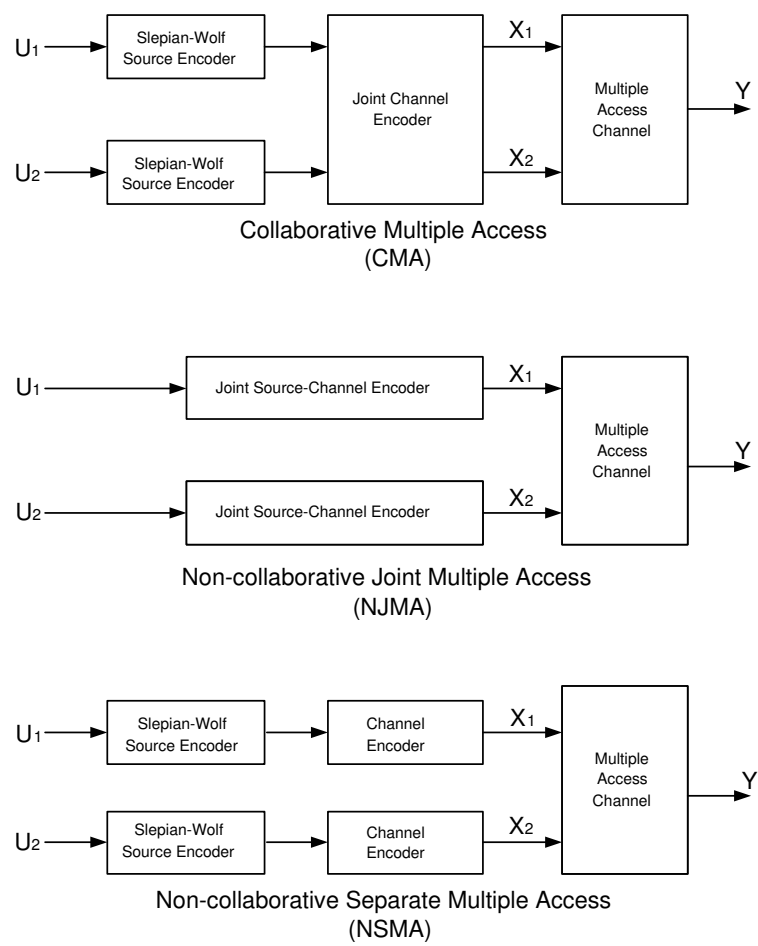

Figure 2: Various multiple access schemes.

input probability distributions, $P_{X_{1} X_{2}}\left(x_{1}, x_{2}\right)$. Denoting the convex hull operation over sets as $\mathcal{C H}($.$) , we have$

$$
\mathbb{R}_{\mathrm{CMA}}=\underset{\forall P_{X_{1} X_{2}}\left(x_{1}, x_{2}\right)}{\mathcal{C H}}\left(\mathbb{L}\left[P_{X_{1} X_{2}}\left(x_{1}, x_{2}\right)\right]\right) .
$$

As the encoders cooperate in this multiple access scheme, we also refer to the CMA capacity as the "cooperative capacity".

NSMA capacity region: In this multiple access scheme, the channel encoders cannot cooperate and have independent inputs which come, for example, from Slepian-Wolf source encoders. Let $P_{X_{1}}\left(x_{1}\right)$ and $P_{X_{2}}\left(x_{2}\right)$ be the distributions on the two independent channel inputs. For any product distribution, $P_{X_{1}}\left(x_{1}\right) P_{X_{2}}\left(x_{2}\right)$, denote the closure of the convex hull of all rate pairs $\left(R_{1}, R_{2}\right)$ satisfying

$$
\begin{array}{r}
R_{1}<I\left(X_{1} ; Y \mid X_{2}\right), R_{2}<I\left(X_{2} ; Y \mid X_{1}\right), \\
R_{1}+R_{2}<I\left(X_{1}, X_{2} ; Y\right),
\end{array}
$$

as $\mathbb{C}\left[P_{X_{1}}\left(x_{1}\right) P_{X_{2}}\left(x_{2}\right)\right]$. The NSMA capacity region, $\mathbb{R}_{\text {NSMA }}$, is the convex hull of the sets $\mathbb{C}\left[P_{X_{1}}\left(x_{1}\right) P_{X_{2}}\left(x_{2}\right)\right]$ over all product input probability distributions, $P_{X_{1}}\left(x_{1}\right) P_{X_{2}}\left(x_{2}\right)$. Hence, we have

$$
\mathbb{R}_{\text {NSMA }}=\underset{\forall\left(P_{X_{1}}\left(x_{1}\right), P_{X_{2}}\left(x_{2}\right)\right)}{\mathcal{C H}}\left(\mathbb{C}\left[P_{X_{1}}\left(x_{1}\right) P_{X_{2}}\left(x_{2}\right)\right]\right) .
$$

Owing to lack of cooperation, the channel encoders cannot increase the correlation between the inputs which 
results in the channel inputs being always independent. This makes the NSMA capacity region an improper ${ }^{1}$ subset of the CMA capacity region since all joint input distributions cannot be generated. As the encoders in this multiple access scheme do not cooperate, we also refer to the NSMA capacity as the "separate capacity".

NJMA capacity region: In this multiple access scheme, there is a single joint source-channel encoder at each transmitter that maps source symbols to channel inputs. The encoders at the two transmitters do not cooperate. This encoder is more general than the combination of the NSMA source and channel encoders, since it can make use of the dependence between the sources to increase the channel mutual information. As the set of channel input distributions that can be generated is larger than that of the NSMA scheme, the NSMA capacity region is an improper subset of the NJMA capacity region. Also, the NJMA capacity region is an improper subset of the CMA capacity region, since the channel encoders cannot generate all joint input probability distributions, owing to lack of coordination. Only those joint input probability distributions that do not require the correlation between channel inputs to be more than the correlation between the source pairs can be generated. Therefore, the set of joint input distributions that can be generated depends on the source that is being transmitted. We denote this set of joint input distributions as $\mathbb{P}_{X_{1} X_{2}}^{J}$.

For any $P_{X_{1} X_{2}}\left(x_{1}, x_{2}\right) \in \mathbb{P}_{X_{1} X_{2}}^{J}$, denote the closure of the convex hull of all rate pairs $\left(R_{1}, R_{2}\right)$ satisfying

$$
\begin{array}{r}
R_{1}<I\left(X_{1} ; Y \mid X_{2}\right), R_{2}<I\left(X_{2} ; Y \mid X_{1}\right), \\
R_{1}+R_{2}<I\left(X_{1}, X_{2} ; Y\right),
\end{array}
$$

as $\mathbb{J}\left[P_{X_{1} X_{2}}\left(x_{1}, x_{2}\right)\right]$. The NJMA capacity region, $\mathbb{R}_{\text {NJMA }}$, is the convex hull of the sets $\mathbb{J}\left[P_{X_{1} X_{2}}\left(x_{1}, x_{2}\right)\right]$ over all joint input probability distributions in $\mathbb{P}_{X_{1} X_{2}}^{J}$. Hence, we have

$$
\mathbb{R}_{\text {NJMA }}=\underset{\forall P_{X_{1} X_{2}}\left(x_{1}, x_{2}\right) \in \mathbb{P}_{X_{1} X_{2}}^{J}}{\mathcal{C H}}\left(\mathbb{J}\left[P_{X_{1} X_{2}}\left(x_{1}, x_{2}\right)\right]\right) .
$$

In this paper, we will also refer to this capacity region as the "Joint source-channel capacity region".

A sufficient criterion for separation to hold: Consider the transmission of a discrete source pair over a multiple access channel. Even if we allow cooperation, there is no hope of transmitting the source pair over the channel with an arbitrarily small error probability unless the Slepian-Wolf source coding region and the CMA capacity region have a non-zero intersection. The question of source-channel separation therefore applies to those source-channel pairs for which these two regions overlap. Hence, for any channel, while considering sourcechannel separation, we always restrict our attention to

\footnotetext{
${ }^{1}$ In this paper an improper subset (superset) of a set $\mathcal{A}$ is defined as a set which is smaller (greater) or equal to $\mathcal{A}$.
}

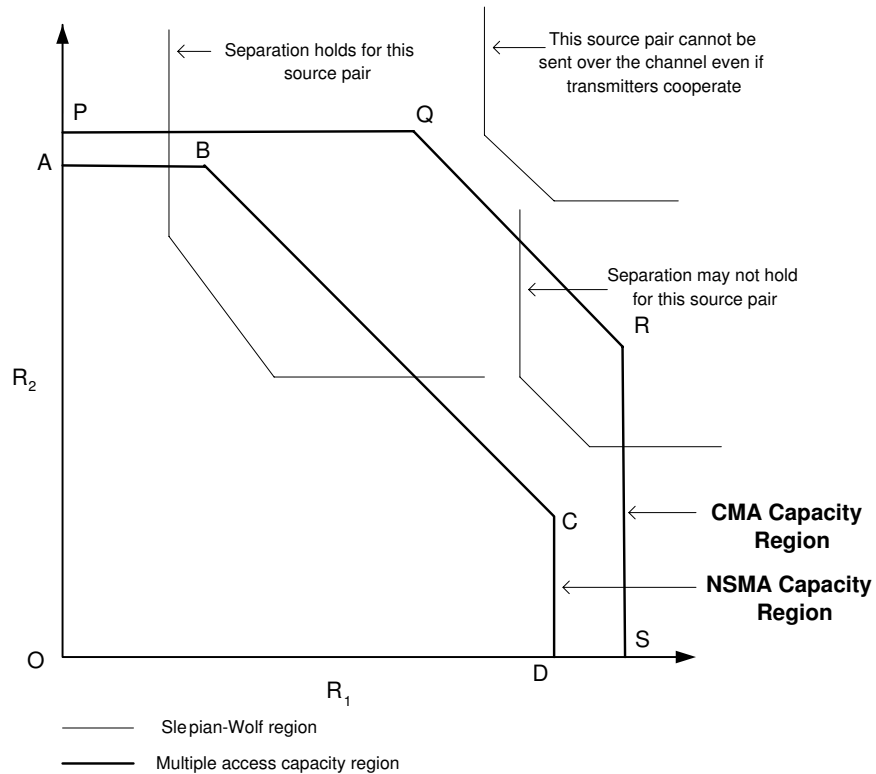

Figure 3: Cooperative and separate multiple access capacity regions.

source pairs whose Slepian-Wolf region overlaps the CMA capacity region of that channel.

If source and channel coding are done separately without coordination between the two transmitters, then only those source pairs for which the Slepian-Wolf region overlaps with the NSMA capacity region can be reliably transmitted over the multiple access channel.

In NJMA, the encoders can make use of the correlation between the sources and hence the NJMA capacity region is an improper superset of the NSMA capacity region. However, since the encoders at the transmitters do not coordinate, this region is an improper subset of the CMA capacity region, in general.

Figure 3 shows the CMA and NSMA capacity regions for a multiple access channel. For ease of illustration, we have considered a multiple-access channel whose capacity regions are pentagons. The regions, in general, may not be pentagons. The Slepian-Wolf regions for three different source pairs are also shown. $A B C D O$ is the NSMA capacity region and $P Q R S O$ the CMA capacity region. Source-channel separation holds for all source pairs whose Slepian-Wolf region overlaps $A B C D O$. For source pairs whose Slepian-Wolf region overlaps only $P Q R S D C B A$, separation may fail. Separation fails for those source pairs for which the NJMA joint source-channel encoder can increase the capacity region beyond the NSMA capacity region so that it intersects the source pair's SlepianWolf source coding region, which allows the source pair to be reliably communicated. The joint source-channel encoders make use of the source statistics to increase correlation between the channel inputs, which may increase the capacity region. More simply, the joint source-channel encoders try to match the source statistics to those needed by the channel to maximize mutual information. How- 


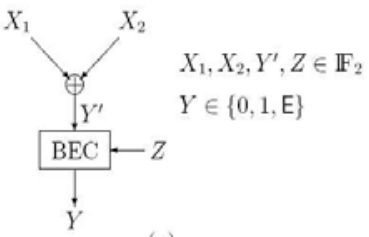

(a)

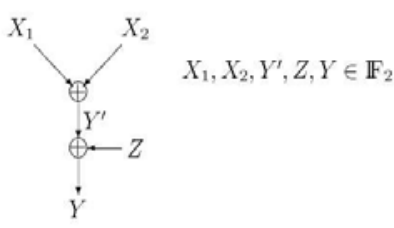

(b)
Figure 4: Binary additive multiple access channels with (a) erasures and (b) additive noise. In both cases, $Z_{1}, Z_{2}, \ldots$ are i.i.d and independent of the channel inputs.

ever, when the joint source-channel encoders cannot increase the capacity region enough to overlap the source pair's Slepian-Wolf region, reliable communication is impossible. In this case we say that separation holds since joint and separate source-channel codes fail equally. Note that the NJMA capacity region cannot increase beyond the CMA capacity region.

Separation fails for the example in [2], since the source pair statistics are perfectly matched to what is required to maximize the channel mutual information. Source pairs whose Slepian-Wolf regions lie outside $P Q R S O$ cannot be reliably transmitted over the channel with or without coordination between the transmitters.

We now derive a sufficient criterion for separation to hold. Since the NJMA capacity region is an improper subset of the CMA capacity region, a sufficient condition for separation to hold for any source-channel pair is that the NSMA capacity region for the channel is the same as its CMA capacity region. For these channels, increasing correlation between the channel inputs does not increase mutual information. Note that for these channels, the region $P Q R S D C B A$ is a null set. We obtain a lemma that states this sufficient criterion for separation to hold for any source-channel pair:

Lemma 1 Separation holds for a multiple access sourcechannel pair if for the channel the following is satisfied

$$
\mathbb{R}_{\text {NSMA }}=\mathbb{R}_{\text {CMA }} .
$$

If the sources are independent, the NJMA joint sourcechannel encoder outputs are always independent. Hence, joint source-channel coding cannot increase the channel mutual information by increasing correlation between the channel inputs, the cause of separation failure. This yields the following lemma:

Lemma 2 Separation holds for a multiple access sourcechannel pair if the sources are independent.

We now consider two additive multiple access channels shown in Figure 4. The first is the additive multiple access channel with erasures and the second is the additive multiple access channel with additive noise. The additive channel with interference only (no channel noise) can be viewed as a special case of either of the noisy models where errors or erasures occur with probability zero. Let $X_{1}$ and $X_{2}$ denote the random channel inputs and use $Y$ to denote the corresponding random channel output. $Y$ equals $X_{1}+X_{2}$ corrupted by erasures in the erasure channel model; we denote the probability of an erasure as $q(1)$. For the additive noise channel model, $Y$ equals $X_{1}+X_{2}+Z$, where $Z$ is the i.i.d additive binary noise. Both examples use addition over the binary field; and noise is independent of the channel inputs.

While we focus on the binary alphabet, results generalize to arbitrary finite fields. The requirement that the finite field be the same for all sources, channel codewords, and additive noise processes cannot, however, be relaxed in general. The channel output alphabet is allowed to differ only in the inclusion of erasures.

Using Lemma 1, we show that source-channel separation holds for binary sources and binary erasure or additive noise multiple access channels where the erasure or additive noise is independent of channel inputs. This result is embodied in the following theorem:

Theorem 1 For any pair of binary sources and any binary erasure or additive noise multiple access channel where the erasure or additive noise is independent of the channel inputs, separation holds. ${ }^{2}$

Proof: Let us first consider the binary multiple access channel shown in Figure 4(a) with erasures that are independent of the inputs. The NSMA capacity (2) is

$$
\mathbb{R}_{\mathrm{NSMA}}^{\text {erasure }}=\left\{\left(R_{1}, R_{2}\right): R_{1}+R_{2}<1-q(1)\right\} .
$$

The three mutual information terms, $I\left(X_{1} ; Y \mid X_{2}\right)$, $I\left(X_{2} ; Y \mid X_{1}\right)$ and $I\left(X_{1}, X_{2} ; Y\right)$, are maximized by uniform distributions for $X_{1}$ and $X_{2}$. For the same channel, the CMA capacity (1) is

$$
\mathbb{R}_{\mathrm{CMA}}^{\text {erasure }}=\left\{\left(R_{1}, R_{2}\right): R_{1}+R_{2}<1-q(1)\right\},
$$

where, the three mutual information terms, $I\left(X_{1} ; Y \mid X_{2}\right)$, $I\left(X_{2} ; Y \mid X_{1}\right)$ and $I\left(X_{1}, X_{2} ; Y\right)$, are maximized by letting $P\left(X_{1}=i, X_{2}=j\right)=\frac{1}{4}$ for $i, j \in\{0,1\}$. Combining $(4$, $5)$, we obtain

$$
\mathbb{R}_{\text {NSMA }}^{\text {erasure }}=\mathbb{R}_{\text {CMA }}^{\text {erasure }}
$$

\footnotetext{
${ }^{2}$ In order to maximize the mutual information between the inputs and output of a multiple access channel with inputindependent noise, we need to maximize the entropy of the channel output. Addition of two independent random variables over the same field corresponds to circular convolution of their probability mass functions (pmfs). If one of the pmfs is uniform, the pmf of the sum is a uniform distribution, which leads to its entropy being maximized. Thus, if the channel inputs are uniformly distributed, they maximize the entropy of the channel output for an additive multiple access channel operating over a finite field. It is this that gives rise to source-channel separation in multiple access networks operating over finite fields.
} 
Hence, by Lemma 1, separation holds. We have thus proved the theorem for the binary multiple access channel with erasures that are independent of the inputs.

Let us now consider the binary multiple access channel shown in Figure 4(b) with noise being independent of the inputs. The NSMA capacity is

$$
\mathbb{R}_{\text {NSMA }}^{\text {add-noise }}=\left\{\left(R_{1}, R_{2}\right): R_{1}+R_{2}<1-H(Z)\right\} .
$$

The three mutual information terms, $I\left(X_{1} ; Y \mid X_{2}\right)$, $I\left(X_{2} ; Y \mid X_{1}\right)$ and $I\left(X_{1}, X_{2} ; Y\right)$, are maximized by uniform distributions for $X_{1}$ and $X_{2}$. For the same channel, the CMA capacity is

$$
\mathbb{R}_{\mathrm{CMA}}^{\text {add-noise }}=\left\{\left(R_{1}, R_{2}\right): R_{1}+R_{2}<1-H(Z)\right\},
$$

where, the three mutual information terms, $I\left(X_{1} ; Y \mid X_{2}\right)$, $I\left(X_{2} ; Y \mid X_{1}\right)$ and $I\left(X_{1}, X_{2} ; Y\right)$, are maximized by letting $P\left(X_{1}=i, X_{2}=j\right)=\frac{1}{4}$ for $i, j \in\{0,1\}$. Combining (6, $7)$, we obtain

$$
\mathbb{R}_{\text {NSMA }}^{\text {add }- \text { noise }}=\mathbb{R}_{\text {CMA }}^{\text {add }- \text { noise }} .
$$

Hence, by Lemma 1, separation holds. We have thus proved the theorem for the binary multiple access channel with additive noise that is independent of the inputs.

The proof of this theorem easily generalizes to the following corollary:

Corollary 1 For any pair of sources defined over a finite field and any erasure or additive noise multiple access channel over the same finite field, where the erasure or additive noise is independent of the channel inputs, separation holds.

We have seen that for channels where the NSMA capacity region is equal to the CMA capacity region, separation holds for all source pairs. The binary multiple access erasure and additive noise channels with input-independent noise are examples. However, when the two regions are not the same, separation may not hold for all source pairs. For the binary additive noise multiple access channel, the CMA and NSMA capacity regions may not be the same when the noise is allowed to depend on the channel inputs and separation may not hold. This lack of separation has been observed earlier by us in [6].

Let us consider the binary additive noise channel model where the additive noise, $Z$, is allowed to depend on the input symbols being transmitted, and has the distribution: $q_{i j}=\operatorname{Pr}\left(Z=1 \mid X_{1}=i, X_{2}=j\right)$ for $i, j \in\{0,1\}$. Hence, a binary additive noise channel can be represented as $b\left(q_{00}, q_{01}, q_{10}, q_{11}\right)$. For any such channel, define the CMA and NSMA sum capacities, $R_{\text {sum }}^{\mathrm{CMA}}\left(q_{00}, q_{01}, q_{10}, q_{11}\right)$ and $R_{\text {sum }}^{\mathrm{NSMA}}\left(q_{00}, q_{01}, q_{10}, q_{11}\right)$, respectively, as

$$
\begin{aligned}
R_{\text {sum }}^{\mathrm{CMA}}\left(q_{00}, q_{01}, q_{10}, q_{11}\right) & \triangleq \max _{\forall P_{X_{1} X_{2}}\left(x_{1}, x_{2}\right)} I\left(X_{1}, X_{2} ; Y\right), \\
R_{\text {sum }}^{\mathrm{NSMA}}\left(q_{00}, q_{01}, q_{10}, q_{11}\right) & \triangleq \max _{\forall\left(P_{X_{1}}\left(x_{1}\right), P_{X_{2}}\left(x_{2}\right)\right)} I\left(X_{1}, X_{2} ; Y\right) .
\end{aligned}
$$

We define the ensemble of all equally likely multiple access binary additive noise channels as

$$
\mathcal{B}=\left\{b\left(q_{00}, q_{01}, q_{10}, q_{11}\right): q_{00}, q_{01}, q_{10}, q_{11} \cup[0,1]\right\},
$$

where, $\mathrm{U}[m, n]$ denotes a uniform probability distribution in $[m, n]$, and $m, n$ are real numbers.

We compute the maximum loss in sum capacity, $\left(R_{\text {sum }}^{\mathrm{CMA}}\left(q_{00}, q_{01}, q_{10}, q_{11}\right)-R_{\text {sum }}^{\mathrm{NSMA}}\left(q_{00}, q_{01}, q_{10}, q_{11}\right)\right)$, over $\mathcal{B}$. We also obtain the expression for the probability that the two sum capacities are unequal for a channel chosen randomly from $\mathcal{B}$. Our results are embodied in the following theorems. The proofs are omitted for brevity.

Theorem 2 For noisy multiple access binary additive noise channels, the maximum difference between the CMA and NSMA sum capacities is $\frac{1}{2}$ bit per channel use.

Theorem 3 The probability that the CMA and NSMA sum capacities are unequal for a channel chosen randomly from $\mathcal{B}$, is $\frac{1}{3}$.

\section{Conclusion}

In this paper, we addressed the topic of separation in multiple access channels. We showed that source-channel separation holds for multiple access channels operating over a common finite field, as long as noise is independent of inputs. For binary multiple access channels with input dependent additive noise, we quantified the loss from failure of separation.

\section{ACKNOWLEDGMENTS}

Siddharth Ray and Muriel Médard acknowledge the research grants NSF CCR-0220039, University of Illinois subaward 02-194 and Hewlett-Packard 008542-008. Michelle Effros acknowledges the support by Caltech's Lee Center for Advanced Networking.

\section{REFERENCES}

[1] T. M. Cover and J. A. Thomas, "Elements of Information Theory", Wiley, 1991.

[2] T. M. Cover, A. El Gamal and M. Salehi, "Multiple access channels with arbitrarily correlated sources", IEEE Transactions on Information Theory, IT-26(6):648-657, November 1980.

[3] H. Liao, "Multiple access channels", Ph.D. Dissertation, Department of Electrical Engineering, University of Hawaii, Honolulu, 1972.

[4] D. Slepian and J. K. Wolf, "Noiseless coding of correlated information sources", IEEE Transactions on Information Theory, IT-19:471-480, 1973.

[5] A. D. Wyner, "Recent Results in the Shannon Theory", IEEE Transcations on Information Theory, 20(1):2-10, January 1974.

[6] M. Effros, M. Médard, T. Ho, S. Ray, D. Karger, R. Koetter and B. Hassibi, "Linear Network Codes: A Unified Framework for Source, Channel, and Network Coding", Advances in Network Information Theory, DIMACS Series in Discrete Mathematics and Theoretical Computer Science, Vol. 66, March 2003, Editors: Gupta et al.

[7] S. S. Pradhan, S. Choi and K. Ramchandran, "Achievable rates for multiple-access channels with correlated messages", pg. 105, ISIT 2004, June 2004. 